\title{
Correction to: Empowering Young Persons During the Transition to Adulthood
}

\author{
Mariela Acuña Mora, Carina Sparud-Lundin, \\ Ewa-Lena Bratt, and Philip Moons
}

\section{Correction to: C. L. Betz, I. T. Coyne (eds.), Transition from Pediatric to Adult Healthcare Services for Adolescents and Young Adults with Long-term Conditions, https://doi. org/10.1007/978-3-030-23384-6_2}

Ms. Mariela Acuña Mora's name was wrongly presented in the original version of the book in Chapter 2. The author's name has now been corrected

The updated online version of this chapter can be found at https://doi.org/10.1007/978-3-030-23384-6_2

\author{
M. Acuña Mora $(\square)$ \\ Institute of Health and Care Sciences, University of Gothenburg, Gothenburg, Sweden \\ KU Leuven Department of Public Health and Primary Care, Leuven, Belgium \\ e-mail: mariela.acuna.mora@gu.se \\ C. Sparud-Lundin \\ Institute of Health and Care Sciences, University of Gothenburg, Gothenburg, Sweden \\ e-mail: carina.s-lundin@fhs.gu.se \\ E.-L. Bratt \\ Institute of Health and Care Sciences, University of Gothenburg, Gothenburg, Sweden \\ Department of Pediatric Cardiology, The Queen Silvia Children's Hospital, \\ Gothenburg, Sweden \\ e-mail: ewa-lena.bratt@gu.se \\ P. Moons \\ Institute of Health and Care Sciences, University of Gothenburg, Gothenburg, Sweden \\ KU Leuven Department of Public Health and Primary Care, Leuven, Belgium \\ Department of Paediatrics and Child Health, University of Cape Town, \\ Cape Town, South Africa \\ e-mail: philip.Moons@med.kuleuven.be
}

